\title{
A rare case: acute ischemic stroke that developed in a case with severe COVID-19 pneumonia
}

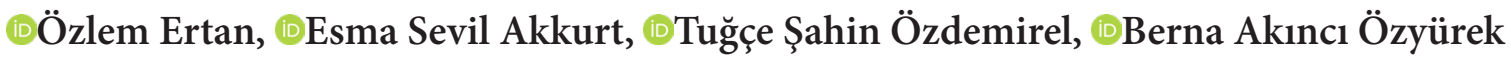

University of Health Sciences Ankara Atatürk Chest Diseases and Chest Surgery Training and Research Hospital, Department of Chest

Disease, Ankara, Turkey

Cite this article as: Ertan Ö, Akkurt ES, Şahin Özdemirel T, Akıncı Özyürek B. A rare case: acute ischemic stroke that developed in a case with severe COVID-19 pneumonia. Anatolian Curr Med J 2021; 3(3); 256-258.

\begin{abstract}
The COVID-19 infection causes involvements in many such as the central nervous system and causes prothrombotic complications. Viral neurotropism, endothelial dysfunction, coagulopathy, and inflammation are suggested mechanisms in the development of acute cerebrovascular disease in COVID-19 patients. The development of a neurological complication is a risk factor for mortality. Patients with high inflammatory markers need to be closely followed-up, because of the risk of the development of complications. It should be kept in mind that there may be neurological involvement in patients with symptoms such as headache, impaired consciousness, vertigo, drowsiness, and loss of strength. In this case, we aimed to present our patient who developed ischemic stroke while under treatment for severe COVID-19 pneumonia.
\end{abstract}

Keywords: COVID-19, pneumonia, ischemic stroke

\section{INTRODUCTION}

Coronavirus disease-2019 (COVID -19) is caused by SARS-COV-2 virus and its systemic and neurological complications are known better today. Characteristically, along with the respiratory system related symptoms (1), neurological symptoms such as headache, impaired consciousness, and vertigo may also develop (2). Especially in the case of severe pneumonia due to COVID-19 infection, the probability of observing neurological symptoms was found to be higher (3). In literature, neurological complications such as acute cerebrovascular disease (CVD), intracranial hemorrhage, seizures, encephalitis, and Guillain Barré syndrome have been reported $(4,5)$. In COVID-19 patients, neurological involvement is a risk factor for mortality (6). We aimed to present a patient in our clinic who developed acute ischemic stroke during follow-up for severe pneumonia.

\section{CASE}

A 62-year-old male patient was admitted to the emergency department with complaints of dyspnea and weakness for two days. Upon admission his vital signs were as fever: $38.2^{\circ} \mathrm{C}$ arterial blood pressure (TA): $120 / 80 \mathrm{mmHg}$, pulse: $89 /$ min, oxygen saturation $82 \%$ at room air. His history revealed he had epilepsy, but he had not used antiepileptic drugs for 3 years and worked as a shoe repairer. On his chest radiography, bilateral increased nonhomogeneous density areas were observed. Laboratory findings were detected as white blood cell $15.910 \times 10^{3} / \mu$, lymphocyte count $0.44 \times 10^{3} / \mu \mathrm{l}, \mathrm{Na}: 127 \mathrm{mEq} / \mathrm{L}, \mathrm{C}$-reactive protein (CRP): $286.82 \mathrm{mg} / \mathrm{L}, \mathrm{D}$-dimer: $0.68 \mathrm{mg} / \mathrm{L}$, ferritin 537.4 $\mathrm{ng} / \mathrm{ml}$ and cardiac troponin was normal. COVID-19 reverse-transcriptase polymerase chain reaction (RT-PCR) test was found positive. Due to the detection of bilateral multilobar ground-glass infiltration and consolidation areas in thoracic computed tomography (CT), the patient was considered as severe pneumonia and was hospitalized in the COVID clinic of our hospital. His hypoxemia regressed with oxygen therapy given by nasal cannula. Dexamethasone, low molecular weight heparin (LMWH) $(1 \times 0.6 \mathrm{ml})$, favipiravir, and nonspecific antibiotherapy were started. On the sixth day of his hospitalization, the loss of strength developed in distal right hand of the patient. On physical examination, he was conscious, and the distal of right upper extremity was weakness and hypoesthesia. During the follow-up of the patient, right central facial paralysis developed and an epileptic seizure for a short duration was observed. The neurology department was 
consulted. On cranial CT, intracranial hemorrhage was not detected. The patient was referred to another hospital with a pre-diagnosis of acute ischemic stroke. From the E-Nabiz system database, it was learned that the cranial diffusion magnetic resonance (MR) imaging taken in the hospital where he was transferred revealed areas showing diffusion restriction in the left cerebral hemisphere and them markers d-dimer (>80 mg/L, reference values: 0-0.5 $\mathrm{mg} / \mathrm{L})$ and IL-6 (10.6 pg/mL) were significantly increased.

\section{DISCUSSION}

COVID-19 infection, which was first defined in December 2019 and affected the whole world, causes many complications. The most common of these are acute respiratory distress syndrome (ARDS), arrhythmia, acute coronary syndrome, and acute renal failure (7). Neurological complications can be observed in hospitalized COVID-19 patients at a rate ranging from $6 \%$ to $36 \%$ (3). In a study of 1683 COVID-19 patients from Spain, the reported CVD rate was $1.4 \%$ (8). Another study found a 2.5 -fold increased risk for the development of CVD in COVID 19 infection (9). It has been shown that neurological symptoms such as headache, impaired consciousness, and paresthesia developed in $36.4 \%$ of patients with COVID-19 (3). Additionally, dizziness, confusion, epileptic seizures, ataxia, anosmia, aging, and muscle pain may be observed in patients (10). Our patient had no neurological symptoms initially when he was admitted, eight days after his positivity, central facial paralysis, epileptic seizure, weakness and hypoesthesia distal of right upper extremity developed due to ischemic stroke. During COVID-19 infection, multiple mechanisms are emphasized in he emergence of these neurological symptoms. In case of severe disease, neurological symptoms may occur as a result of cerebral hypoxia due to respiratory failure (11). Besides, central nervous system (CNS) invasion due to trans-synaptic spread by the involvement of the olfactory neurons and epithelium is another proposed mechanism (5). In brain biopsies taken from patients with a diagnosis of COVID 19 who developed CVD, thrombotic microangiopathy and endothelial damage have been shown (8). Severe COVID-19 patients may be at risk of thrombogenesis and cerebral ischemia because of both biochemical hypercoagulability and direct vascular endothelial damage (12). In addition to direct CNS involvement, the systemic inflammatory response that occurs with SARS-Cov-2 infection may disrupt the blood-brain barrier and cause peripheral cytokines to pass into the CNS (13). Characteristically, COVID-19 coagulopathy manifests itself with significantly increased d-dimer levels, mild thrombocytopenia, and prolonged prothrombin time (10). Hyperactivation of inflammatory factors causes d-dimer and platelet abnormalities by disrupting the coagulation system (2). Increased IL-6 as a response to systemic inflammation has been associated with an increased risk of $\operatorname{CVD}(14,15,16)$. In the studies have, it has been shown that there are higher d-dimer and CRP levels in COVID-19 patients in whom CVD developed than patients whom it did not (17). Also, patients with severe CNS involvement present with higher blood urea nitrogen levels as well as lower lymphocyte and platelet counts, while laboratory findings may not be useful in patients with peripheral nervous system involvement or patients with non-severe CNS involvement (11). Our patient did not have a significant increase in the d-dimer levels upon admission, however, the CRP value was significantly higher. After the development of CVD, d-dimer (>80 mg/L) and IL-6 (10.6 pg/ml) values were observed to increase significantly.

Besides observing that cerebral ischemia due to COVID-19 was a risk factor for mortality, it has also been found that COVID-19 patients who had an acute ischemic stroke during infection had significantly lower survival rates compared to those who did not (6).

\section{CONCLUSION}

SARS-CoV-2 infection is related to prothrombotic events. It should be kept in mind that in patients with high inflammatory markers, persistent headache, newly developed impaired consciousness and conditions such as agitation and paresthesia may be signs of neurological involvement.

\section{ETHICAL DECLARATIONS}

Informed Consent: Written informed consent was obtained from all participants who participated in this study.

Referee Evaluation Process: Externally peer-reviewed.

Conflict of Interest Statement: The authors have no conflicts of interest to declare.

Financial Disclosure: The authors declared that this study has received no financial support.

Author Contributions: All of the authors declare that they have all participated in the design, execution, and analysis of the paper, and that they have approved the final version.

\section{REFERENCES}

1. Epidemiology Working Group for NCIP Epidemic Response, Chinese Center for Disease Control and Prevention. Zhonghua Liu Xing Bing Xue Za Zhi 2020; 41: 145-51.

2. Niazkar HR, Zibaee B, Nasimi A, Bahri N. The neurological manifestations of COVID-19: a review article. Neurol Sci 2020; 41: 1667-71. 
3. Mao L, Wang M, Chen S, et al. Neurological manifestations of hospitalized patients with COVID-19 in Wuhan, China: a retrospective case series study. JAMA Neurol 2020; 77: 683-90.

4. Bridwell R, Long B, Gottlieb M. Neurologic complications of COVID-19. Am J Emerg Med 2020; 38: 1549.e3-1549.e7.

5. Bodro M, Compta Y, Sánchez-Valle R. Presentations and mechanisms of CNS disorders related to COVID-19. Neurol Neuroimmunol Neuroinflamm 2020; 8: e923.

6. Annie F, Bates MC, Nanjundappa A, Bhatt DL, Alkhouli M. Prevalence and outcomes of acute ischemic stroke among patients $\leq 50$ years of age with laboratory confirmed COVID-19 infection. Am J Cardiol 2020; 130: 169-70.

7. Vakili K, Fathi M, Pezeshgi A, et al. Critical complications of COVID-19: A descriptive meta-analysis study. Rev Cardiovasc Med 2020; 21: 433-42.

8. Hernández-Fernández F, Sandoval Valencia H, Barbella-Aponte RA, et al. Cerebrovascular disease in patients with COVID-19: neuroimaging, histological and clinical description. Brain 2020; 143: 3089-103.

9. Aggarwal G, Lippi G, Michael Henry B. Cerebrovascular disease is associated with an increased disease severity in patients with coronavirus disease 2019 (COVID-19): a pooled analysis of published literature. Int J Stroke 2020; 15: 385-9.

10. Tsivgoulis G, Palaiodimou L, Zand R, et al. COVID-19 and cerebrovascular diseases: a comprehensive overview. Ther Adv Neurol Disord 2020; 13: 1756286420978004.

11. Azhideh A. COVID-19 Neurological manifestations. Int Clin Neurosci J 2020; 7; 2: 54.

12. Goldberg MF, Goldberg MF, Cerejo R, Tayal AH. Cerebrovascular disease in COVID-19. Am J Neuroradiol 2020; 41; 1170-2.

13. Romero-Sánchez CM, Díaz-Maroto I, Fernández-Díaz E, et al. Neurologic manifestations in hospitalized patients with COVID-19: The ALBACOVID registry. Neurology 2020; 95: 1060-70.

14. Reddy ST, Garg T, Shah C, et al. Cerebrovascular disease in patients with COVID-19: a review of the literature and case series. Case Rep Neurol 2020; 12; 199-209.

15. Wu Y, Xu X, Chen Z, et al. Nervous system involvement after infection with COVID-19 and other coronaviruses. Brain Behav Immun 2020; 87: 18-22.

16. Wang F, Nie J, Wang $\mathrm{H}$, et al. Characteristics of lymphocyte subsets and cytokines in peripheral blood of 123 hospitalized patients with 2019 novel coronavirus pneumonia (NCP). MedRxiv 2020: 2020.02.10.20021832.

17. Li Y, Li M, Wang M, et al. Acute cerebrovascular disease following COVID-19: a single center, retrospective, observational study. Stroke Vasc Neurol 2020; 5: 279-84. 